\title{
RADIATION AND HEAT GENERATION EFFECTS ON A CONVECTIVE FLOW THROUGH A POROUS MEDIUM WITH PERIODIC TEMPERATURE BOUNDARY CONDITION
}

\author{
Alabison Raimi M. \\ Department of Statistics, \\ The Federal Polytechnic, Ede, \\ Osun State, Nigeria.
}

\author{
Olaleye Olalekan A. \\ Department of Statistics \\ The Federal Polytechnic, Ede, \\ Osun State, Nigeria.
}

\author{
Bamigboye Jonah S. \\ Department of Statistics \\ The Federal Polytechnic, Ede, \\ Osun State, Nigeria.
}

\begin{abstract}
In this work, the effects of thermal radiation and heat generation on a free convective flow of an incompressible fluid through a porous medium with a periodic surface temperature and first order chemical reaction is investigated. Rosseland approximation was adopted to describe the radiative heat flux. The heat generation is linearly dependent on the temperature. The dimensionless coupled nonlinear partial differential equations were reduced to ordinary differential equations and solved analytically using asymptotic technique. The effects of various parameters apart from radiation and heat generation terms in the governing equation on velocity, temperature and concentration were analyzed in details and shown graphically, as well as with tables. Velocity increases with increase in the heat generation parameter, and decreased with the radiation parameter, $R$, as well as the Prandtl number.
\end{abstract}

Keywords- Convective flow, Heat Generation, Periodic temperature boundary condition, Porous medium, Radiation.

\section{INTRODUCTION}

Heat and mass flow of fluid through a porous medium has attracted a tremendous attention of researchers over the years. This is largely due to its significant applications in Engineering and Industries. Examples of such application can be found in geothermal and oil reserviour applications. Transport phenomena of fluid with low porosity are described by Darcy law which relates the flow velocity to the pressure gradient across the medium linearly.

Several literatures abound on Transport phenomena of fluids in porous medium. Sandeep et al. (2012) studied the effects of radiation and chemically reaction of unsteady heat and mass flow of viscous fluid over a semi-infinite vertical plate through a parabolic surface subject to variable suction velocity. Umavathi (2015) considered the combined effect of temperature-dependent viscosity and thermal conductivity on Double-Diffusive Convection flow of a permeable fluid. ElAziz (2009) analysed the flow and heat transfer over an unsteady stretching sheet. Yih (1999) investigated the effects of radiation on the flow of fluid through a porous media. He considered the flow through a cylindrical surface. Ahmed et al (2013) investigated the effects of cross-diffusion, chemical reaction heat radiation and viscous dissipation on exponentially stretching surface.

Mohammed (2013) presented his work on the influence of unsteady free convective flow of fluid through a highly porous medium in the presence of thermal radiation, heat generation and chemical reaction. A parabolic surface temperature was considered.

In view of the above investigations, heat and mass flow of a viscous incomprehensible fluid through a porous medium is considered in the present work. The flow is made to pass over a horizontal surface with a periodic temperature and the suction velocity is time-dependent. The governing partial differential equations obtained with their boundary conditions constituted a boundary valued problem (BVP). These were solved analytically using perturbation method. The results were discussed with the aid of graphs and tables.

\section{MATHEMATICAL FORMULATION}

The flow is convective unsteady and two dimensional. The fluid is viscous and incomprehensible flowing through a porous medium under the influence of thermal radiation, heat generation and chemical reaction of first order. Radiation is considered only from the fluid.

All the properties of the fluid are assumed to be constant except three density variation with temperature and concentration as described by Boussinesq's approximation in 


\section{International Journal of Engineering Applied Sciences and Technology, 2019 \\ Vol. 4, Issue 5, ISSN No. 2455-2143, Pages 108-113 \\ Published Online September 2019 in IJEAST (http://www.ijeast.com)}

the body force term. With the above assumptions the governing equations are:

$$
\frac{\partial v^{\prime}}{\partial y^{\prime}}=0
$$

$\frac{\partial u^{\prime}}{\partial t^{\prime}}+v^{\prime} \frac{\partial u^{\prime}}{\partial y^{\prime}}=-\frac{1}{\rho} \frac{\partial P^{\prime}}{\partial x^{\prime}}+\vartheta \frac{\partial^{2} u^{\prime}}{\partial y^{\prime 2}}+g \beta\left(T^{\prime}-T_{\infty}^{\prime}\right)+g \beta^{*}\left(C^{\prime}-C_{\infty}^{\prime}\right)-\frac{\vartheta}{K^{\prime}} \varphi u^{\prime}$

$\sigma \frac{\partial T^{\prime}}{\partial t^{\prime}}+\varphi v^{\prime} \frac{\partial T^{\prime}}{\partial y^{\prime}}=\frac{k}{\rho C_{p}} \frac{\partial^{2} T^{\prime}}{\partial y^{\prime 2}}-\frac{\varphi}{\rho C_{p}} \frac{\partial q_{r}}{\partial y^{\prime}}+\frac{Q_{0}}{\rho C_{p}}\left(T^{\prime}-T_{\infty}^{\prime}\right)$

$\frac{\partial C^{\prime}}{\partial t^{\prime}}+v^{\prime} \frac{\partial C^{\prime}}{\partial y^{\prime}}=D \frac{\partial^{2} C^{\prime}}{\partial y^{\prime 2}}-K_{r}^{\prime}\left(C^{\prime}-C_{\infty}^{\prime}\right)$

Subject to:

$u^{\prime}=U_{p}^{\prime}, T^{\prime}=T_{w}^{\prime}+A^{\prime} \cos \omega^{\prime} t^{\prime}, C^{\prime}=C_{w}^{\prime}$, at,$y^{\prime}=0$

$u^{\prime} \rightarrow U_{\infty}^{\prime}, T^{\prime} \rightarrow T_{\infty}^{\prime}, C^{\prime} \rightarrow C_{\infty}^{\prime}$, as,$y^{\prime} \rightarrow \infty$

Equation (1) indicates that the suction velocity normal to the plate is either a constant or a function of time, $t$.

That is,

$v^{\prime}=-V_{0}$ or $v^{\prime}=-V_{0}\left(1+\varepsilon A e^{i \omega^{\prime} t^{\prime}}\right)$

But for the purpose of this work, as used by Seshaiah et al. [7] and Nwaigwe (2010) we consider:

$$
v^{\prime}=-V_{0}\left(1+\varepsilon A e^{i \omega^{\prime} t^{\prime}}\right)
$$

The negative sign shows that the suction is towards the plate and $\omega^{\prime}$ is the frequency of oscillation. A and $\varepsilon$ are very small positive parameters such that $\varepsilon A<<1$. That is $\varepsilon A$ is a perturbation parameter.

Outside the boundary layer, equation (2) gives:

$\frac{1}{\rho} \frac{d P^{\prime}}{d x^{\prime}}=-\frac{\varphi \vartheta}{K^{\prime}} V_{\infty}^{\prime}$

Using Rosseland approximation as reported by Salem (2012), the radiative heat flux term is given as:

$q_{r}=\frac{-4 \sigma_{s} \partial T^{\prime 4}}{3 K_{e} \partial y^{\prime}}$
Applying the Taylor's series about $T_{\infty}^{\prime}$ and neglecting higher order terms, the above equation becomes

$T^{\prime 4} \cong 4 T_{\infty}^{\prime 3} T-3 T_{\infty}^{\prime 4}$

Thus the above equations of the heat flux term becomes

$q_{r}=\frac{-16 \sigma_{s} T_{\infty}^{\prime 3}}{3 K_{e}}$

Introducing the following dimensionless quantities as in Mohammed (2013),

$$
\begin{aligned}
& u=\frac{u^{\prime}}{U_{\infty}^{\prime}}, \quad y=\frac{V_{0} y^{\prime}}{v}, \quad U_{p}=\frac{U_{p}^{\prime}}{U_{\infty}^{\prime}}, \quad t=\frac{t^{\prime} V_{0}^{2}}{v}, \quad \lambda=\frac{\sigma}{\varphi}, \\
& \theta=\frac{T^{\prime}-T_{\infty}^{\prime}}{T_{w}^{\prime}-T_{\infty}^{\prime}}, \quad C=\frac{C^{\prime}-C_{\infty}^{\prime}}{C_{w}^{\prime}-C_{\infty}^{\prime}} \quad G r=\frac{\vartheta g \beta\left(T_{w}^{\prime}-T_{\infty}^{\prime}\right)}{U_{\infty}^{\prime} V_{0}^{2}}, \\
& G c=\frac{\vartheta g \beta^{*}\left(C_{w}^{\prime}-C_{\infty}^{\prime}\right)}{U_{\infty}^{\prime} V_{0}^{2}}, \quad K=\frac{K^{\prime} V_{0}^{2}}{\varphi \vartheta^{2}}, \quad P_{r}=\frac{\rho C_{p} \varphi v}{k} \\
& R=\frac{K_{e} k}{4 \varphi \sigma_{s} T_{\infty}^{3}}, \quad Q=\frac{Q_{0} \vartheta}{\varphi \rho C_{p} V_{0}^{2}}, \quad S c=\frac{\vartheta}{D}, \quad K_{r}=\frac{K_{r}^{\prime} \vartheta}{V_{0}^{2}}
\end{aligned}
$$

Substituting equations (7) - (10) into equations (2) - (6) reduces the governing equations to the following dimensionless forms:

$\frac{1}{4} \frac{\partial u}{\partial t}-\left(1+\varepsilon A e^{i \omega t}\right) \frac{\partial u}{\partial y}=\frac{\partial^{2} u}{\partial y^{2}}+G r \theta+G c C+\frac{1}{K}(1-u)$

$\frac{1}{4} \lambda \frac{\partial \theta}{\partial t}-\left(1+\varepsilon A e^{i \omega t}\right) \frac{\partial \theta}{\partial y}=\frac{1}{N_{r}} \frac{\partial^{2} u}{\partial y^{2}}+Q \theta$

$\frac{1}{4} \frac{\partial C}{\partial t}-\left(1+\varepsilon A e^{i \omega t}\right) \frac{\partial C}{\partial y}=\frac{1}{S c} \frac{\partial^{2} u}{\partial y^{2}}-K_{r} C$

Subject to:

$u=U_{p}, \theta=1+A^{*} \cos \omega t, C=1$, at $y=0$

$u \rightarrow 1, \theta \rightarrow 0, C \rightarrow 0$, as $y \rightarrow \infty$

where,

$$
N_{r}=\left(1-\frac{4}{3 R+4}\right) P_{r}
$$

\section{ANALYTICAL SOLUTION OF THE PROBLEM}

The resulting equations (11) - (13) are coupled nonlinear partial differential equations with their boundary conditions in equations (14) - (15) were reduced to ordinary differential equations and solved analytically. This is done by adopting the 


\section{International Journal of Engineering Applied Sciences and Technology, 2019 \\ Vol. 4, Issue 5, ISSN No. 2455-2143, Pages 108-113 \\ Published Online September 2019 in IJEAST (http://www.ijeast.com)}

following solutions for the velocity, temperature and concentration of the fluid respectively:

$$
\begin{aligned}
& U(y, t)=U_{0}(y)+\varepsilon A e^{i \omega t} U_{1}(y)+\varepsilon^{2} A^{2} e^{2 i \omega t} u_{2}(y)+\cdots \\
& \theta(y, t)=\theta_{0}(y)+\varepsilon A e^{i \omega t} \theta_{1}(y)+\varepsilon^{2} A^{2} e^{2 i \omega t} \theta_{2}(y)+\cdots \\
& C(y, t)=C_{0}(y)+\varepsilon A e^{i \omega t} C_{1}(y)+\varepsilon^{2} A^{2} e^{2 i \omega t} C_{2}(y)+\cdots
\end{aligned}
$$

Since $\varepsilon A \ll 1$, higher order terms of $\varepsilon A$ can be neglected. The assumed solutions in equation (16) become;

$U(y, t)=U_{0}(y)+\varepsilon A e^{i \omega t} U_{1}(y)$

$\theta(y, t)=\theta_{0}(y)+\varepsilon A e^{i \omega t} \theta_{1}(y)$

$C(y, t)=C_{0}(y)+\varepsilon A e^{i \omega t} C_{1}(y)$

Substituting equation (17) into equation (11) - (13) and compiling terms in the order of $(\varepsilon \mathrm{A})^{0}$ and $\varepsilon \mathrm{A}$, we obtain

$\frac{\partial^{2} u_{0}}{\partial y^{2}}+\frac{\partial u_{0}}{\partial y}-\frac{1}{K} u_{0}=-\frac{1}{K}-G r \theta_{0}-G c C_{0}$

$\frac{\partial^{2} u_{1}}{\partial y^{2}}+\frac{\partial u_{1}}{\partial y}-\left(\frac{1}{4} i \omega+\frac{1}{K}\right) u_{1}=-G r \theta_{1}-G c C_{1}-\frac{\partial u_{0}}{\partial y}$

$\frac{\partial^{2} \theta_{0}}{\partial y^{2}}+N_{r} \frac{\partial \theta_{0}}{\partial y}+N_{r} Q \theta_{0}=0$

$\frac{\partial^{2} \theta_{1}}{\partial y^{2}}+N_{r} \frac{\partial \theta_{1}}{\partial y}+N_{r}\left(Q-\frac{1}{4} \lambda i \omega\right) \theta_{1}=-N_{r} \frac{\partial \theta_{0}}{\partial y}$

$\frac{\partial^{2} C_{0}}{\partial y^{2}}+S c \frac{\partial C_{0}}{\partial y}-S c K_{r} C_{0}=0$

$\frac{\partial^{2} C_{1}}{\partial y^{2}}+S c \frac{\partial C_{1}}{\partial y}-S c\left(K_{r}+\frac{1}{4} i \omega\right) C_{1}=-S c \frac{\partial C_{0}}{\partial y}$

Equations (15) are subject to

$U_{0}=U_{p} \quad \theta_{0}=1+A^{*} \cos \omega t \quad C_{0}=1$

$U_{1}=0 \quad \theta_{1}=0 \quad C_{1}=0 \quad$ at $\quad y=0$

$U_{0} \rightarrow 1 \quad \theta_{0} \rightarrow 0 \quad C_{0} \rightarrow 0$

$U_{1} \rightarrow 0 \quad \theta_{1} \rightarrow 0 \quad C_{1} \rightarrow 0 \quad$ as $\quad y \rightarrow \infty$

By solving equation (18) subject to the corresponding boundary conditions in equation (19) we obtain the solution for the velocity, temperature and concentration profiles as represented in equation (17) respectively as follows;

$$
\begin{aligned}
U(y, t)= & 1+A_{1} e^{m_{6} y}+A_{2} e^{m_{2} y}+A_{3} e^{m_{10} y}+ \\
& {\left[\left(1+A^{*} \cos \omega t\right)\left(A_{4} e^{m_{6} y}-A_{4}^{*} e^{m_{8} y}\right)+A_{6} e^{m_{6} y}\right.} \\
& \left.+A_{8} e^{m_{10} y}+A_{9} e^{m_{12} y}+A_{10} e^{m_{2} y}+A_{11} e^{m_{4} y}\right] \varepsilon A e^{i \omega t} \\
\theta(y, t)= & \left(1+A^{*} \cos \omega t\right)\left[e^{m_{6} y}+N_{2}\left(e^{m_{6} y}-e^{m_{8} y}\right) \varepsilon A e^{i \omega t}\right]
\end{aligned}
$$

$C(y, t)=e^{m_{2} y}+N_{1}\left(e^{m_{2} y}-e^{m_{4} y}\right) \varepsilon A e^{i \omega t}$

Where,

$$
\begin{aligned}
& m_{2}=-\frac{1}{2}\left[S_{c}+\sqrt{ }\left(S_{c}^{2}+4 S_{c} K_{r}\right)\right] \\
& m_{4}=-\frac{1}{2}\left[S_{c}+\sqrt{ }\left(S_{c}^{2}+4 S_{c}\left(\frac{1}{4} i \omega+k_{r}\right)\right)\right] \\
& m_{6}=-\frac{1}{2}\left[N_{r}+\sqrt{N_{r}^{2}-4 N_{r} Q}\right] \\
& m_{8}=-\frac{1}{2}\left[N_{r}+\sqrt{N_{r}^{2}+4 N_{r}\left(\frac{1}{4} \lambda i \omega-Q\right)}\right] \\
& \left.m_{10}=-\frac{1}{2}[1+\sqrt{(1+4 / k})\right] \\
& m_{12}=-\frac{1}{2}\left[1+\sqrt{ }\left(1+4\left(\frac{1}{4} i \omega+\frac{1}{k}\right)\right)\right. \\
& \mathrm{A}_{1}=\frac{-G_{\mathrm{r}}}{m_{\mathrm{G}}^{\mathrm{n}}+m_{\mathrm{E}}-\frac{1}{k}} \quad \mathrm{~A}_{2}=\frac{-G_{\mathrm{c}}}{m_{2}^{2}+m_{\mathrm{n}}-\frac{1}{k}} \\
& \mathrm{~A}_{4}=\frac{-G_{\mathrm{Y}} N_{2}}{m_{6}^{2}+m_{6}-\left(\frac{1}{4} i \omega+\frac{1}{2}\right)} \\
& \mathrm{A}_{3}=U_{p}-\left[1+\mathrm{A}_{1}\left(1+\mathrm{A}^{*} \cos \omega t\right)+\mathrm{A}_{2}\right] \\
& \mathrm{A}_{4}^{*}=\frac{-G_{\mathrm{r}} N_{2}}{m_{\mathrm{g}}^{2}+m_{\mathrm{g}}-\left(\frac{1}{4} i \omega+\frac{1}{2}\right)} \\
& \mathrm{A}_{5}=\frac{-G_{c} N_{1}}{m_{2}^{2}+m_{2}-\left(\frac{1}{4} i \omega+\frac{1}{k}\right)} \\
& A_{5}^{*}=\frac{-G_{e} N_{1}}{m_{4}^{2}+m_{4}-\left(\frac{1}{4} i \omega+\frac{1}{k}\right)} \\
& A_{6}=\frac{-m_{6} A_{1}}{m_{6}^{2}+m_{6}-\left(\frac{1}{4} i \omega+\frac{1}{k}\right)} \\
& \mathrm{A}_{7}=\frac{-m_{2} \mathrm{~A}_{2}}{m_{2}^{2}+m_{\mathrm{z}}-\left(\frac{1}{4} i \omega+\frac{1}{2}\right)} \\
& \mathrm{A}_{\mathrm{g}}=\frac{-m_{10} \mathrm{~A}_{8}}{m_{10}^{2}+m_{10}-\left(\frac{1}{4} i \omega+\frac{1}{k}\right)} \\
& A_{9}=-\left(\left(1+A^{*} \cos \omega t\right)\left(A_{4}-A_{4}^{*}\right)+\left(A_{5}-A_{5}^{*}\right)+A_{6}+A_{7}+A_{8}\right) \\
& \mathrm{A}_{10}=\mathrm{A}_{2}+\mathrm{A}_{7} \quad \mathrm{~A}_{11}=-\mathrm{A}_{\mathrm{5}}^{8}
\end{aligned}
$$

\section{RESULTS AND DISCUSSION}

The numerical results of the transient velocity, temperature and concentration gotten from the previous section were computed and displayed on graphs and tables. The effects of 


\section{International Journal of Engineering Applied Sciences and Technology, 2019 \\ Vol. 4, Issue 5, ISSN No. 2455-2143, Pages 108-113 \\ Published Online September 2019 in IJEAST (http://www.ijeast.com)}

the physical parameters that emerged namely; the Prandtl number $\left(\mathrm{P}_{\mathrm{r}}\right)$, radiation parameter $(\mathrm{R})$, internal heat generation parameter $(\mathrm{Q})$, thermal Grashof number $(\mathrm{Gr})$, Solutal Grashof number (Gc), permeability of the porous medium (K), Schmidt number $(\mathrm{Sc})$, Chemical reaction parameter $\left(\mathrm{K}_{\mathrm{r}}\right)$ were examined on the velocity, temperature, concentration, Nusset number and Skin-friction.

The following parametric default values were adopted in the computation except otherwise stated:

$$
\begin{aligned}
& G r=2.0, G c=2.0, K=5.0, \lambda=1.4, S c=0.2, \\
& R=5.0, K_{r}=2.0, Q=0.1, P_{r}=0.71, U_{p}=0.4, \\
& A=0.5, t=1.0, \varepsilon=0.01, A_{0}=1.0 \text { and } \omega=\frac{\pi}{2}
\end{aligned}
$$

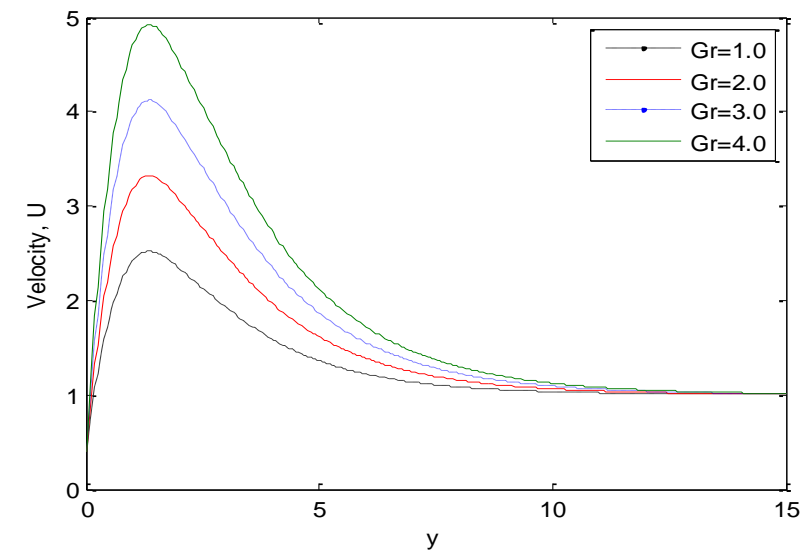

Fig. 1: Velocity profile for different values of $\mathrm{Gr}$

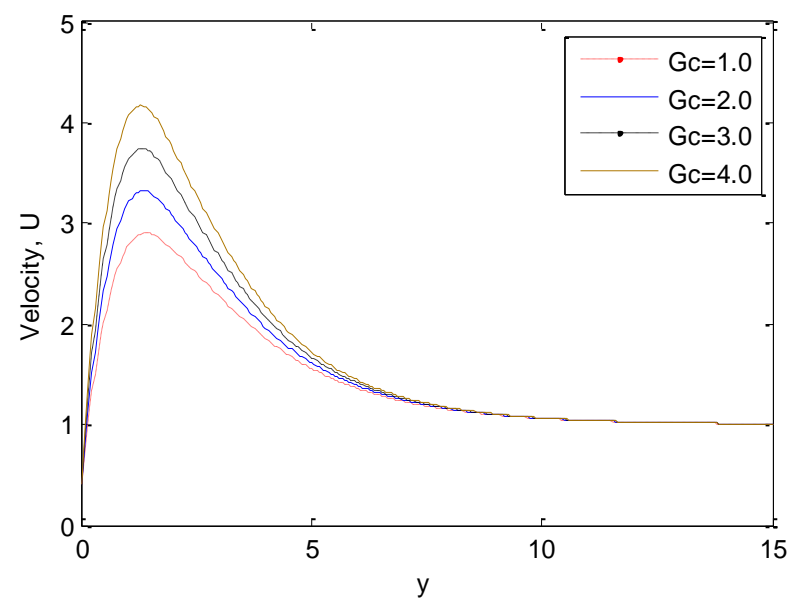

Fig. 2: Velocity profile for different values of Gc

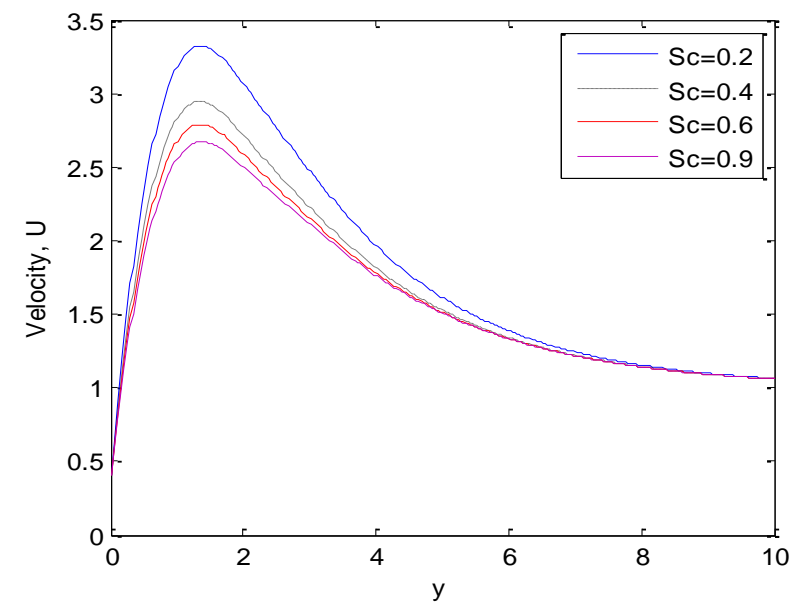

Fig. 3: Velocity profile for different values of Sc

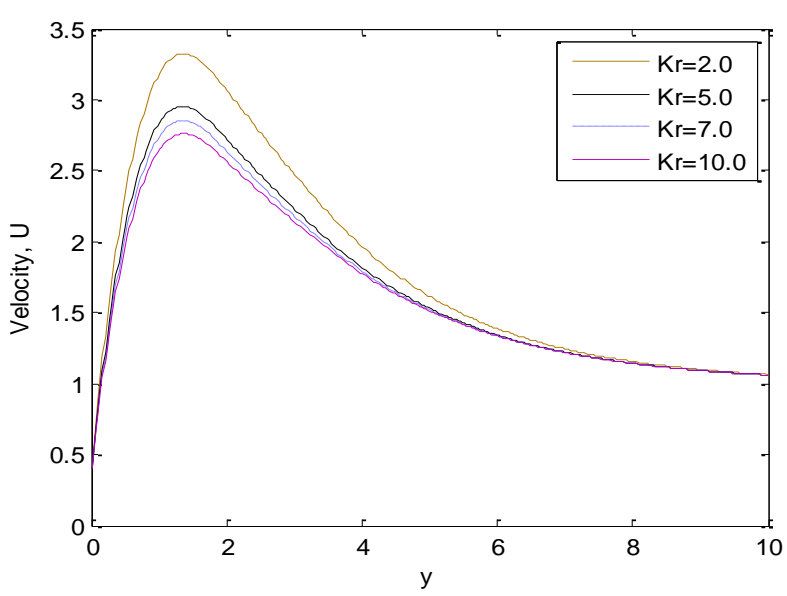

Fig. 4: Velocity profile for different values of $K_{r}$

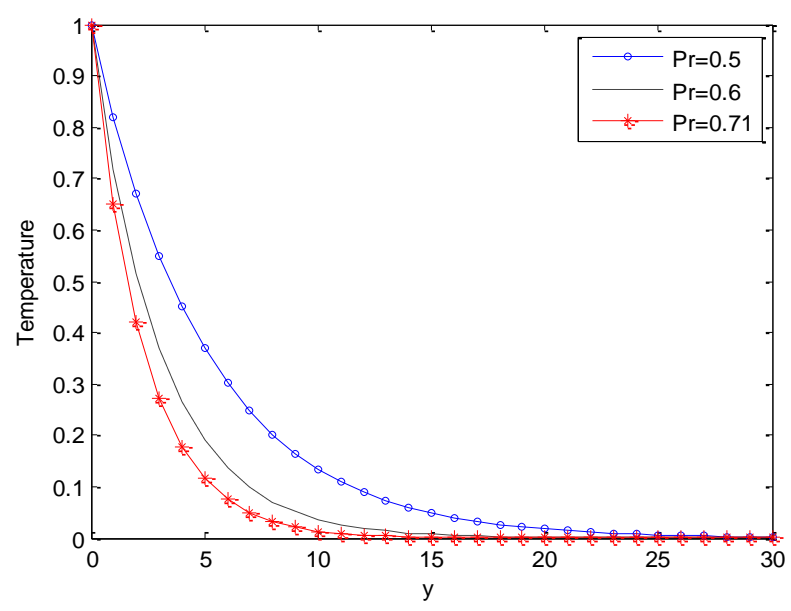

Fig. 5: Temperature profile for different values of $P_{r}$ 


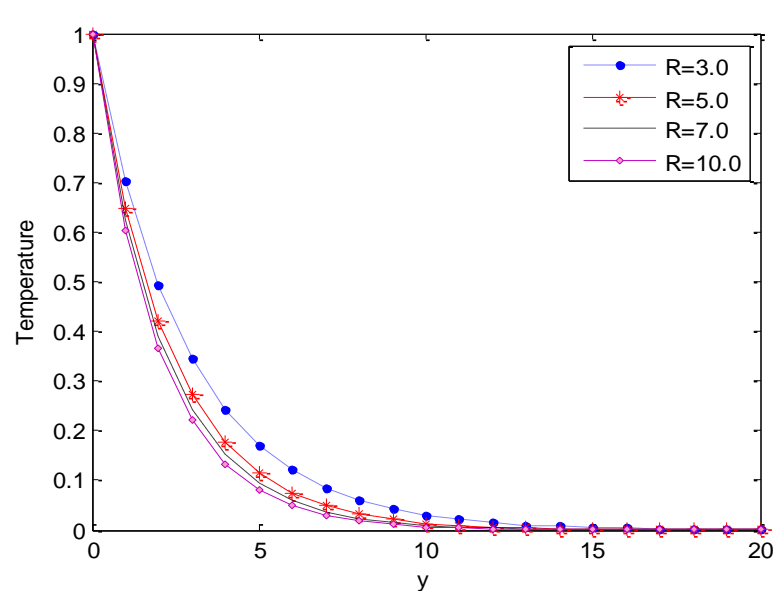

Fig. 6: Temperature profile for different values of $\mathrm{R}$

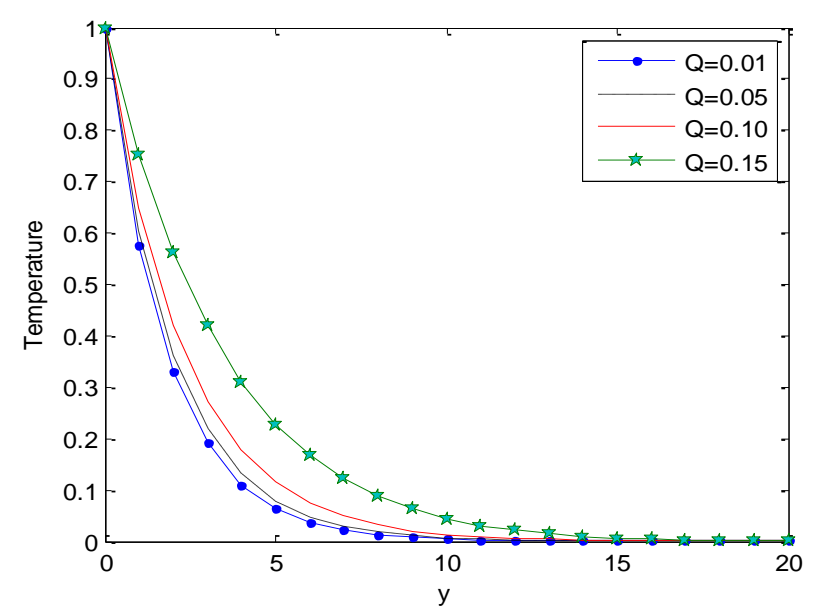

Fig. 7: Temperature profile for different values of $\mathrm{Q}$

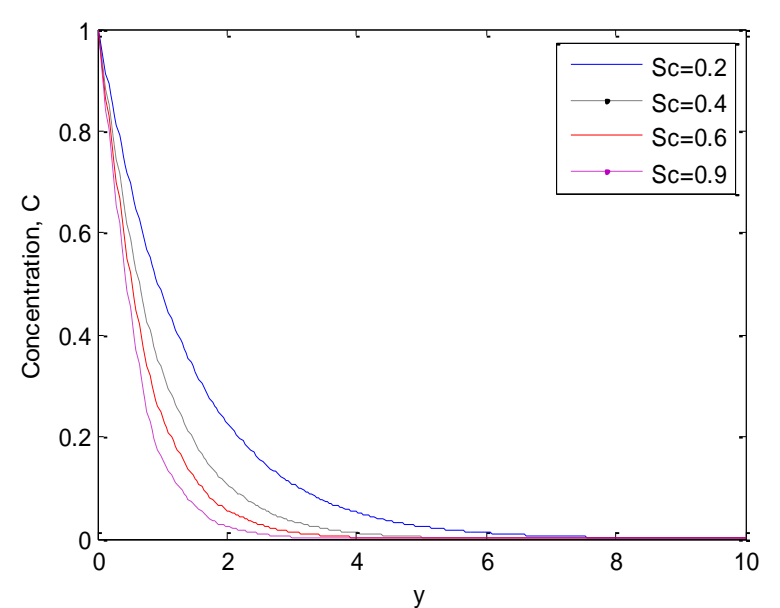

Fig. 8: Concentration profile for different values of Sc

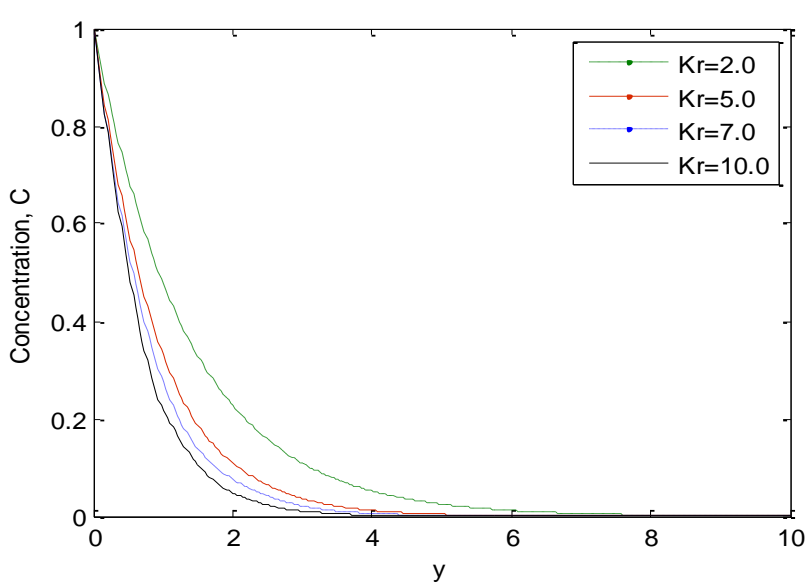

Fig. 9: Concentration profile for different values of $\mathrm{K}_{\mathrm{r}}$

According to the results obtained and displayed on graphs above,

- The velocity of the fluid

- increased with increase in thermal Grashof number and solutal Grashof number (figs. 1 \& 2)

- decreased with increasing Schmit number and chemical reaction parameter (figs. $3 \&$ 4)

- The temperature of the fluid

- decreased as the radiation parameter increased (fig. 6)

- decreased at the boundary layer with the increase in Prandtl number (fig. 5)

- increased with increasing heat generation (fig. 7)

The concentration decreased with increase in both Schmit number and the chemical reaction parameter (figs. $8 \& 9$ )

\section{CONCLUSION}

This work has investigated the radiation and heat generation effects on a convective flow through a porous medium with periodic temperature boundary condition. According to the results gotten, when the radiation and thermal buoyancy forces are applied at an increasing rate, there is corresponding increase in the temperature of the fluid and the rate of its flow also speeds up. Thus, to raise the temperature of the fluid and increase the rate of its flow, the two parameters mentioned are of great importance. Moreover, the concentration can be reduced when the chemical reaction parameter and the Schmit number are increased.

\section{REFERENCE}

1. Ahmed A. Khidir and Precious Sibanda (2013). "Cross-Diffusion, Viscous Dissipation and Radiation Effects on an Exponentially Stretching Surface in Porous Media". Mass Transfer-Advances in 
Sustainable Energy and Environment Oriented Numerical Modeling, Chapter 5. http/dx.doi.org/10.5772/55320

2. El-Aziz M. A. (2009). "Radiation Effects on the Flow and Heat Transfer over an Unsteady Stretching Sheet". International Communications in Heat and Mass Transfer, 36 (pp. 521-524).

3. Mohammed Ibrahim S. (2013). "Radiation Effects on Mass Transfer Flow through a Highly Porous Medium with Heat Generation and Chemical Reaction", ISRN Computational Mathematics.

4. Nwaigwe C. (2010). "Mathematical Modeling of Ground Temperature with Suction Velocity and Radiation." American Journal of Scientific and Industrial Research, (pp.238-241).

5. Salem Fathy A. (2012). "Effects of Variable Properties on MHD Heat and Mass Transfer Flow Near a Stagnation Point Towards a Stretching Sheet in a Porous Medium with Thermal Radiation," Chinese Physical Society and IOP Publishing Ltd.

6. Sandeep N., Vijaya Bhaskar Reddy A. and Sugunamma V. (2012). "Effects of Radiation and Chemical Reaction on Transient MHD Free Convective Flow over a Vertical Plate Through Porous Medium". Chemical and Process Engineering Research, Vol.2. ISSN 2225-0913. www.iiste.org.

7. Seshaiah B., V. S. (2103) "The Effects of Chemical Reaction and Radiation on Unsteady MHD free Convective Fluid Flow Embedded in a Porous Medium with TIme-Dependent Suction with Temperature Gradient Heat Source," International Journal of Scientific Knowledge, Vol. 3, Issue 2, (pp. 12-24).

8. Umavathi J. C. (2015). "Combined Effects of Variable Viscosity and Variable Thermal Conductivity on Double-Diffusive Convection Flow of a Permeable Fluid in a Vertical Channel. Transp. Porous Med. 108 (pp. 659-678). DOI 10.1007/s11242-015-0494-9

9. Yih K. A. (1999). "Radiation Effects on Natural Convection over a Vertical Cylinder Embedded in Porous Media". International Communications in Heat and Mass Transfer, Vol. 26, Issue 2 (pp.259267.). 